\title{
Cyclins A, B, E and p27 in Endometrial Endometrioid Adenocarcinoma
}

\author{
SIMI SANTALA ${ }^{1}$, ANNE TALVENSAARI-MATTILA ${ }^{1}$, YLERMI SOINI ${ }^{2,3}$, \\ PAULA KUVAJA ${ }^{2}$ and MARKKU SANTALA ${ }^{1}$ \\ Departments of ${ }^{1}$ Obstetrics and Gynecology and ${ }^{2}$ Pathology, Oulu University Hospital, Oulu, Finland; \\ ${ }^{3}$ Department of Pathology and Forensic Medicine, Institute of Clinical Medicine, Pathology and Forensic Medicine, \\ School of Medicine, Cancer Centre of Eastern Finland, University of Eastern Finland, Kuopio, Finland
}

\begin{abstract}
Background/Aim: We have previously shown that cyclin $A, B$ and $E$ hold prognostic significance in endometrial endometrioid adenocarcinoma. The aim of this study was to investigate the impact of cyclin-dependent kinase inhibitor p27 on cancer-specific survival and other clinicopathological variables, as well as further analyze the relationship between $p 27$ and cyclins $A, B$ and $E$ and their combined relation to prognosis in the disease. Patients and Methods: The study comprised of 211 patients surgically treated for endometrial endometrioid adenocarcinoma at the Oulu University Hospital between 1992 and 2000. Tissue samples were immunohistochemically stained for cyclins A, $B$ and $E$, as well as p27. Clinicopathological data were retrospectively retrieved from the patients' records. Results: In this study, universally low cyclin expression was found to be an independent, favorable prognostic factor in endometrial endometrioid adenocarcinoma. A strong correlation was found between cyclin $A$ and cyclin $B$ expression and weaker correlations between other cyclin and p27 pairs. Nuclear p27 expression correlated with stage and produced near-significant results in univariate survival analysis. Conclusion: Combining the expression level of different cyclins may be useful in determining the prognosis in endometrial cancer. Unfortunately, it remains unclear whether high p27 expression is a poor or a favorable prognostic factor. Further large-scale studies are required to assess the effects of cyclins and p27 in endometrial cancer.
\end{abstract}

Correspondence to: Markku Santala, Department of Obstetrics and Gynecology, Oulu University Hospital, P.O. Box 24, 90029 Oulu, Finland. Tel: +358 83153586, Fax: +358 83154310, e-mail: markku.santala@oulu.fi

Key Words: p27, cyclins, CDKs, endometrial adenocarcinoma, prognosis.
Cyclin-dependent kinases (CDKs) are a family of enzymes that play a key role in the regulation of the cell cycle. The activity of CDKs is regulated by the binding of an activator from the cyclin family and by inhibition of CDKs and cyclin-CDK complexes by cyclin-dependent kinase inhibitors (CKIs). By binding to their specific CDKs, cyclins A, B, D and $\mathrm{E}$ directly influence cell cycle progression. Cyclin DCDK4/CDK6 and cyclin E-CDK2 complexes control $\mathrm{G}_{1}$ and $\mathrm{S}$ phase progression. Cyclin B-CDK1 complex regulates the transition between $G_{2}$ and $M$ phase. Cyclin $A$, on the other hand, has specificity for both CDK1 and CDK2 and, along with cyclins $B$ and $E$, influences both $S$ phase progression and $\mathrm{G}_{2} / \mathrm{M}$ transition (1-2).

CKIs have been divided into two classes: the inhibitors of CDK4 (INK4) p15, p16, p18 and p19 and kinase inhibitor proteins (Kips) p21, p27 and p57. INK4s inhibit CDK4 and CDK6, whereas Kips have a more dualistic role; they inhibit CDK2-cyclin complexes but they also seem to facilitate the binding of cyclin D to CDK4/6 (3).

Understandably, the direct involvement of cyclins, CDKs and their inhibitors in the cell cycle has given rise to interest in cancer research. Deregulation of different cyclins, CDKs and their inhibitors have been shown to hold prognostic significance in neoplasms of different origin $(2,4)$. However, their involvement in endometrial cancer is only partially understood.

Endometrial cancer is the leading gynecological malignancy in Western countries. In Finland, the ageadjusted incidence and mortality rates in 2012 were 12.9 and 2.2 per 100,000 women, respectively (5). Endometrial cancer has traditionally been divided into type I and type II tumors. Type I tumors are typically of endometrioid subtype and thought to be associated with estrogen excess, obesity, hormone-receptor positivity and endometrial hyperplasia. Type II tumors comprise of subtypes, such as serous and clear-cell carcinoma, which develop in an atrophic endometrium (6). The most common histopathological type, 
endometrioid adenocarcinoma, carries a five-year survival rate of $80 \%$ and over (7). Despite the relatively favorable prognosis, there is a need to find novel predictive and prognostic factors to ensure more aggressive treatment, where necessary.

Previously, we have analyzed the impact of cyclin A, cyclin B and cyclin E on endometrial endometrioid adenocarcinoma (8-9). In this study, we evaluate the effects of p27 expression on conventional clinicopathological and prognostic factors in the same patient population and further analyze the correlations between cyclins A, B and E, as well as $\mathrm{p} 27$, and their relation to prognosis.

\section{Patients and Methods}

Endometrial adenocarcinoma samples were obtained from 211 patients treated at the Department of Obstetrics and Gynecology of Oulu University Hospital, Oulu, Finland, between 1992 and 2000. The median age of the patients was 64 years (range=37-98) and median body mass index (BMI) $29.7 \mathrm{~kg} / \mathrm{m}^{2}$ (range=19.8-49.1). Extrafascial hysterectomy, bilateral salpingo-oophorectomy and pelvic lymphadenectomy were the operative treatments in most cases $(n=206)$. Two patients had preoperative, 134 patients postoperative and two pre- and postoperative radiotherapy. Four patients received neoadjuvant and 45 adjuvant cisplatin-based chemotherapy.

All cases were staged according to the International Federation of Gynecology and Obstetrics (FIGO) classification 1988 and, for the purpose of this series of studies, accurately converted to FIGO classification 2009. Stage I tumors were present in 140, stage II in 30 , stage III in 36 and stage IV in five patients. Histopathological examination revealed grade 1 in 112, grade 2 in 66 and grade 3 in 33 of the samples. Median follow-up time was 77 months (range $=0$ 136). At the end of the follow-up, 53 of the 211 patients had died; 33 patients of the disease, 20 of other causes. The number of patients with all four biomarkers analyzed was 199 .

Approval for the study was obtained from the Regional Ethics Committee of the Northern Ostrobothnia Hospital District.

Immunohistochemical staining. Representative tumor-containing paraffin blocks were selected and $4-\mu \mathrm{m}$ sections were cut for immunohistochemistry. Slides were deparaffinized in xylene, followed by a declining alcohol series. Prior to adding the antibody, the samples were incubated in Tris-EDTA (Sigma-Aldrich, St. Louis, MO, USA) ( $\mathrm{pH} \mathrm{9.0)} \mathrm{and} \mathrm{boiled} \mathrm{in} \mathrm{a} \mathrm{microwave} \mathrm{oven} \mathrm{for}$ $2 \mathrm{~min}$ at $850 \mathrm{~W}$ and for $15 \mathrm{~min}$ in $350 \mathrm{~W}$. Endogenous peroxidase activity was blocked by using $0.1 \%$ hydrogen peroxide in methanol solution (Envision-kit; Dako, Glostrup, Denmark).

The slides were incubated for $1 \mathrm{~h}$ with a mouse monoclonal p27 antibody (Leica Biosystems; Newcastle upon Tyne, UK) at a 1:200 dilution, followed by a standard avidin-biotin complex protocol using Envision staining kit (Dako). The sections were counterstained with hematoxylin and mounted with overslipping film TissueTek (Sakura, Torrance, CA, USA). Instead of antibody, phosphate-buffered saline was used as a negative control and known positive endometrial carcinoma samples from prior series were used as positive controls. p27 positivity was evaluated as the percentage of positive nuclear staining/whole tumor area in the section. Other staining patterns were evaluated separately (8-10).
Statistical analyses. All statistical analyses were carried out by using the SPSS for Mac version 21 software (International Business Machines Corp, Armonk, NY, USA). The relationships between clinicopathological variables and nuclear p27 were assessed with the Kruskal-Wallis or Mann-Whitney $U$-test. Chi-squared test was used to analyze the relationships between clinicopathological variables and cytoplasmic p27. Correlations between continuous variables were tested by Spearman's rank correlation. Receiver operator characteristic curve (ROC) was used to determine the accuracy of p27 as a discriminator between patients with a good and poor prognosis over a range of cut-off points. Cumulative survival was analyzed by Kaplan-Meier analysis. The differences between the subgroups were compared by means of a log-rank test. The Cox proportional hazards model was used in multivariate analysis to assess the independency of the prognostic factors.

\section{Results}

The median labeling index (LI) of nuclear p27 was $10 \%$ (range $=0-100$ ). Forty-seven cases had negative staining and seven were not analyzed due to a limited number of tumor sections (Figure 1).

In both grade 1 and grade 2 tumors, the median LI was $10 \%$ (range $=0-100)$. An increase to $17.5 \%$ (range $=0-100)$ was seen in grade 3 tumors. The differences were not statistically significant. Combined early stages (I+II) had a lower median LI of $10 \%$ (range $=0-100$ ) compared to the median LI of $17.5 \%$ (range=3-100) in advanced stages (III+IV). The difference was statistically significant (Table I).

Correlation analyses between cyclin A, cyclin B, cyclin E and $\mathrm{p} 27$ revealed a strong correlation between cyclin $\mathrm{A}$ and cyclin B (Figure 2) and weaker correlations between other cyclin pairs or p27 (Table II).

For survival analyses, a cut-off value of $7.5 \%$ for $\mathrm{p} 27$ was estimated from the ROC curve and the patients were grouped accordingly to low- (LI $\leq 7.5 \%$ ) and high- (LI >7.5\%) expression groups. The same method was utilized to group cyclin A, cyclin B and cyclin E expression, as described previously (8-10). In cumulative survival analysis, the patients with a low p27 LI had a five-year cancer-specific survival rate of $90 \%$, compared to a survival rate of $81 \%$ for patients with a high LI. The difference, however, did not reach statistical significance (Figure $3 \mathrm{~A}$ ).

Cytoplasmic p27 staining was considered separately but its expression did not correlate with grade or stage. Cumulative survival analysis showed a slight tendency towards poorer survival in patients with intense cytoplasmic p27 staining but the results were not statistically significant (data not shown).

Cox proportional hazard model was used to assess the independency of different variables. A controlled analysis was run with first fitting the model with FIGO stage, grade and cyclin $\mathrm{A}$, as this was estimated to best fit the data according to previously published approaches (8). In the second phase, cyclin B, cyclin E and nuclear p27 were individually added but none was able to improve the model. 


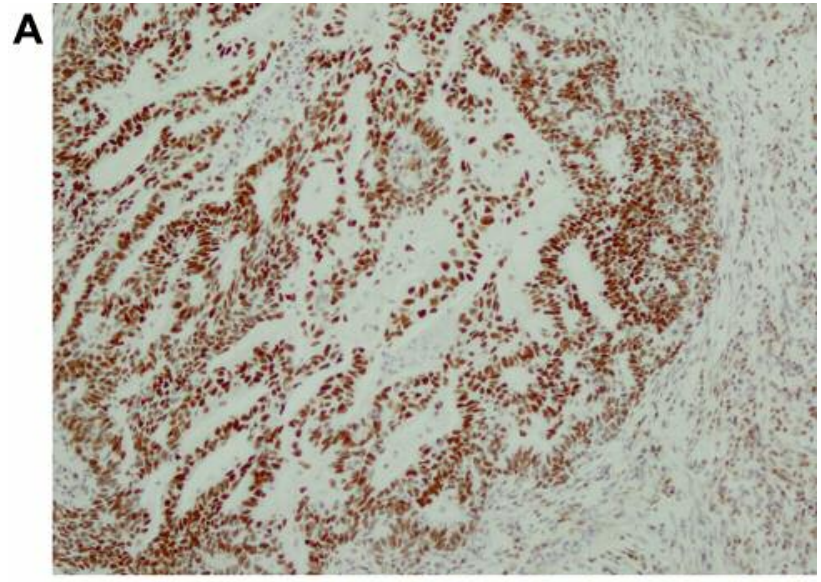

B

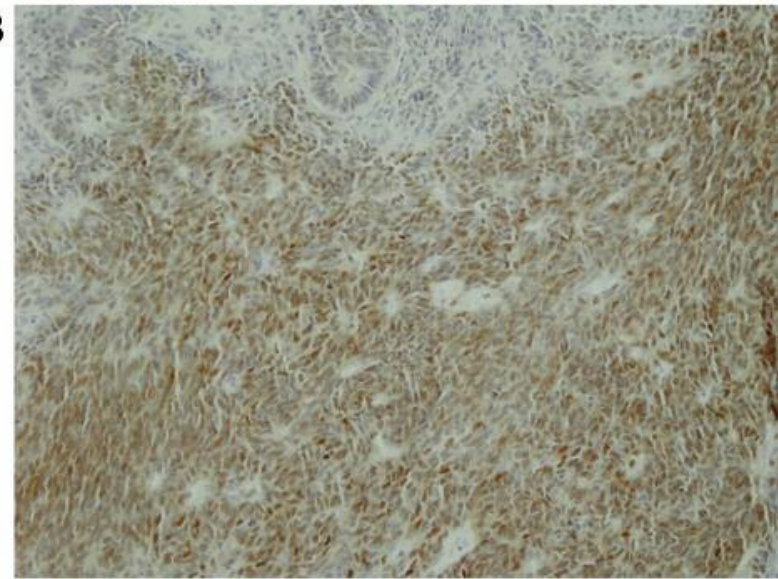

C

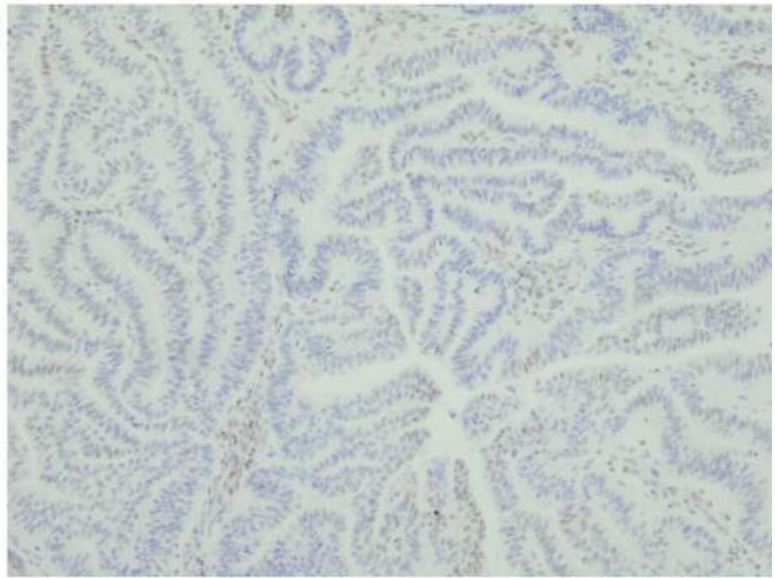

Figure 1.p27 staining. A, Strong positive nuclear staining for $p 27 . B$, Partly cytoplasmic and nuclear staining pattern for p27. C, Negative staining for $p 27$.

All the cyclins and p27 were analyzed as categorical variables based on the cut-off value-defined groups.

Splitting the data according to the cut-off value-defined groups revealed that cyclin A was a poor prognostic factor

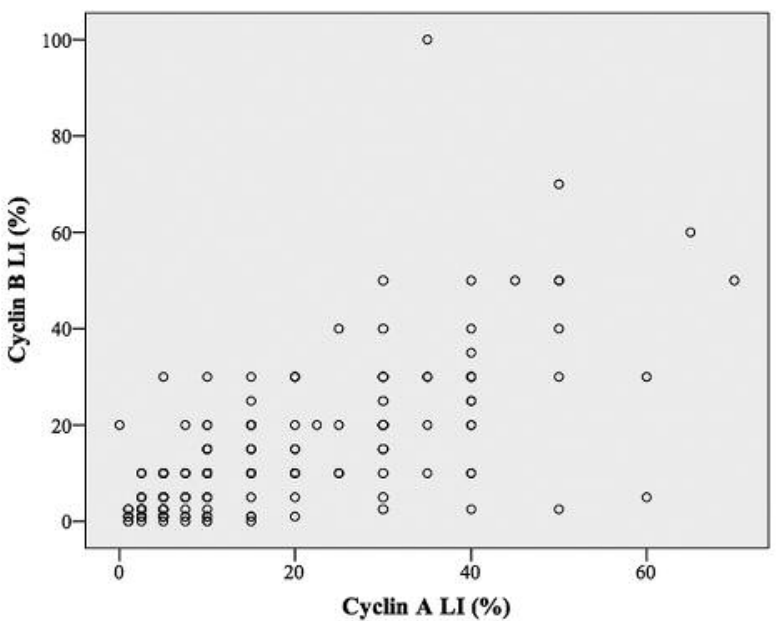

Figure 2. Expression of cyclin $A$ and cyclin B showing a strong correlation.

Table I. Labeling indexes (LI) of stage.

\begin{tabular}{lccc}
\hline Stage & Number of patients & LI median $(\%)$ & $p$-Value \\
\hline I+II & 164 & $10(0-100)$ & \\
III+IV & 40 & $17.5(3-100)$ & $0.027^{*}$ \\
\hline
\end{tabular}

*Stage I + stage II vs. stage III + stage IV.

Table II. Correlation coefficients between cyclin A, cyclin B and cyclin E, as well as $p 27$.

\begin{tabular}{llll}
\hline & Cyclin A & Cyclin B & Cyclin E \\
\hline Cyclin B & $0.694^{*}$ & & \\
Cyclin E & $0.345^{*}$ & $0.305^{*}$ & \\
p27 & $0.275^{*}$ & $0.185^{*}$ & $0.147^{* *}$ \\
\hline
\end{tabular}

${ }^{*} p<0.01 ; * * p<0.05$.

in univariate analysis in a subset of patients with a low cyclin B expression and vice versa. Based on this notion, we combined the groups in which either of the cyclins showed high expression and compared the survival to the group in which both cyclins showed low expression. Cumulative survival analysis revealed a five-year cancer-specific survival rate of $95 \%$ for patients with a low expression of both cyclins, compared to a $76 \%$ survival rate for patients with either cyclin showing high expression (Figure 3B). In the Cox proportional hazard model with stage and grade, the 

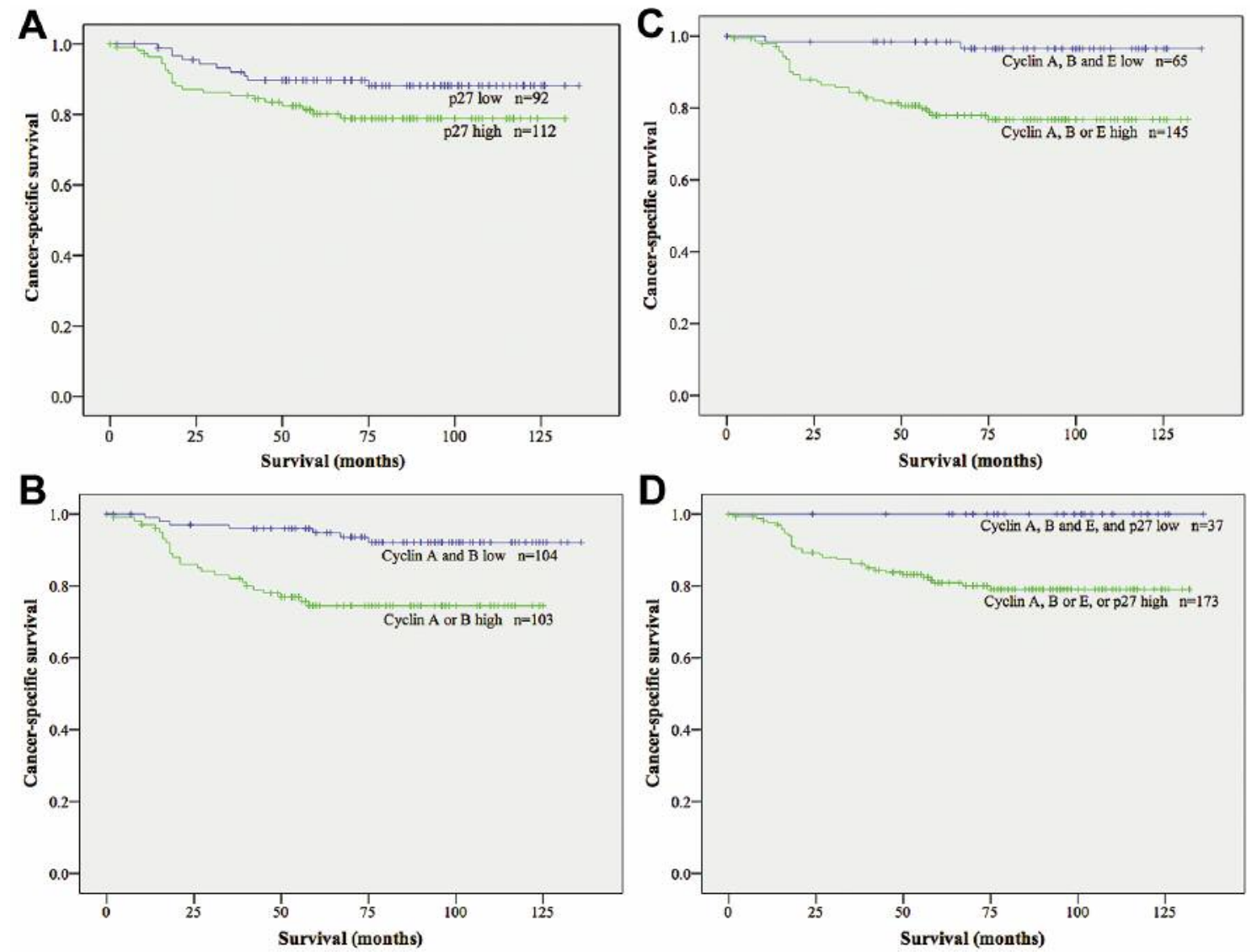

Figure 3. Cancer-specific survival. A, Cumulative survival according to $p 27$ as defined by the cut-off value of 7.5\% ( $p=0.075$; at 60 months $p=0.069$ ). $B$, Cumulative survival according to combined cyclin $A$ and cyclin $B(p<0.001$; at 60 months $p<0.001)$. C, Cumulative survival according to universally low cyclin expression ( $p=0.001$; at 60 months $p<0.001)$. Cyclin $A$, cyclin B and cyclin E cut-off value each $12.5 \%$. D, Cancer-specific survival reached $100 \%$ when cyclin $A$, cyclin B, cyclin E and p27 expressions were universally low and $81 \%$ if any of the markers showed high expression ( $p=0.004)$. Crosses indicate censored cases.

group in which either cyclin showed high expression was estimated as having a hazard ratio of $3.2(95 \%$ confidence interval $(\mathrm{CI})=1.3-8.0 ; p=0.013)$.

Similarly, there was a tendency towards poorer survival in patients with low cyclin A and B expression but high cyclin E expression. Cumulative survival analysis in the group with a universally low cyclin expression showed a five-year cancer-specific survival rate of $98 \%$, compared to a $79 \%$ survival rate for the group in which any of the cyclins showed high expression (Figure 3C). Multivariate analysis in the Cox proportional hazard model was then performed with the following factors: high expression of any cyclin, FIGO stage and grade (Table III).

Notably, when all four biomarkers showed low expression, cancer-specific survival reached $100 \%$ (Figure 3D). However, the number of patients was not sufficient to analyze further the effects of combining all four biomarkers.
Table III. Independent and significant prognostic factors and hazard ratios of cancer-specific death.

\begin{tabular}{lcc}
\hline Variable & Hazard ratio & $p$-Value to remove \\
\hline Stage & & $<0.001$ \\
I & 1 & \\
II & $2.6(1.0-6.9)$ & \\
III & $3.8(1.7-8.5)$ & \\
IV & $15(4.0-55)$ & \\
Grade & & \\
1 & 1 & \\
2 & $2.1(0.88-5.0)$ & 0.019 \\
3 & $3.7(1.5-9.3)$ & \\
Cyclin expression & & \\
all $\leq 12.5$ & 1 & \\
any $>12.5$ & $5.8(1.4-25)$ & \\
\hline
\end{tabular}




\section{Discussion}

p27 was first identified as an inhibitor of cell proliferation, more specifically a cyclin-dependent kinase inhibitor. As one might expect, low expression of p27 has been linked to poor prognosis in a number of malignant neoplasms, including gastric, lung and breast cancer (11). However, the current understanding of its effects is more dualistic in nature: p27 is an inhibitor of CDK2 but also seems to facilitate the activation of CDK4 and CDK6. Interestingly, whereas nuclear p27 staining has been shown to be beneficial for patient outcome in a number of malignancies, cytoplasmic p27 staining seems to convey a more ominous message. Based on these findings, it has been suggested that cellular localization, mediated by the phosphorylation of Thr-157, Thr-198 or Ser-10 by Akt or other enzymes, is an important part in determining the inhibitory/activating effects of p27 $(3,4)$.

In this study, the impact p27 had on cancer-specific survival produced only near-significant findings. Interestingly, however, a tendency towards poorer survival was observed in patients with a high nuclear p27 expression.

Likewise, previous studies have struggled to find a clear correlation between p27 expression and prognosis (12-16). Previously, Seeber et al. (16) showed that high p27 expression is a poor prognostic factor in multivariate analysis but only in a subset of patients with perinecrotic hypoxiainducible factor $1 \alpha$ (HIF-1 $\alpha$ ) expression. Furthermore, Dellas et al. (13) showed that combined loss of p27 and phosphatase and tensin homologue (PTEN) carried a more favorable prognosis in multivariate analysis. Interestingly, however, in two large-scale studies by Steinbakk et al. (1718), a correlation between low p27 expression and poor prognosis was found in univariate analysis in the curettages of early stage (FIGO I and I-II) tumors. Overall, survival analyses have produced mixed results and it remains unclear whether nuclear p27 expression should be regarded as a poor or favorable prognostic factor in endometrial cancer.

Contrary to the vast majority of studies, we also separately considered cytoplasmic p27 expression but did not find an association with survival or other variables. In accordance with our findings, Nycum et al. (12) found no correlation between cytoplasmic p27 staining and survival or other clinicopathological variables. Mutations of the gene encoding p27 are thought to be extremely rare in malignant tumors, whereas other mechanisms, particularly cytoplasmic mislocalisation, have been suggested to account for the role of p27 in tumorigenesis (4). Our findings do not support this mechanism in endometrial cancer.

We previously reported that cyclin A is an independent prognostic factor in endometrial endometrioid adenocarcinoma (8), whereas cyclins B (9) and E (10) are prognostic factors only in univariate analysis. In this study, we further analyzed the relationship between the cyclins and found that a universally low expression is associated with a more favorable cancer-specific survival in multivariate analysis.

We also found a strong correlation between cyclin A and cyclin B and weak to moderate correlations between other cyclin pairs or $\mathrm{p} 27$. Some correlations between cyclins have previously been analyzed in endometrial cancer (19-21); however, no studies have reported a strong correlation between cyclin A and cyclin B, although reports on other malignant neoplasms have found a strong correlation between them (22-24). These findings may be attributable to the fact that both cyclin A and cyclin B are expressed in the cell cycle roughly at the same time, share transcription promoters and proteolysis machinery and may, thus, to some extent, reflect similar changes in cell proliferation (25). In this sense, combining cyclin A and cyclin B in survival analyses may be useful, as shown in this study.

In the present series, we found a correlation between nuclear p27 expression and advanced stage, but not with grade. Previously, Nycum et al. (12) reported a correlation between combined nuclear and cytoplasmic p27 expression and stage. However, there is only one study by Watanabe et al. (26) reporting a correlation between nuclear p27 expression and stage. Along with others, they also found a correlation between p 27 and grade $(13,16,27)$. On the other hand, divergent findings also exist (14-15, 28). The divergence of findings may be explicable in terms of various immunohistochemical stainings, interpretation techniques and data grouping methods used.

The patients in this study underwent surgery during a time when pelvic lymphadenectomy was routinely performed, which may have had a positive effect on the accuracy of staging. All patients were treated in the same facility, Oulu University Hospital, and all samples treated with a uniform protocol. Immunohistochemical stainings were analyzed by two investigators blinded to the clinical data. Systematic surgical staging was performed in accordance with the FIGO (2009) criteria. Follow-up was organized systematically and was sufficiently long to show deaths from the disease.

The disadvantages of this work include the retrospective study setting and the subjectivity of evaluating immunohistochemical staining. Furthermore, a small number of patients received chemo- and/or radiotherapy for their tumors prior to surgery, possibly affecting the p27 and cyclin levels in some samples. Many patients also received postoperative therapy, as was the standard treatment at the time. The benefit of such treatment has since been re-evaluated and it is currently not recommended for low-risk patients (29). The effects of the treatment on patient survival have likely been minimal, thus not affecting the integrity of the study.

In conclusion, we have shown here that universally low cyclin expression is an independent, favorable prognostic factor in endometrial endometrioid adenocarcinoma. 
Furthermore, we found a correlation between nuclear p27 expression and advanced stage. Nuclear p27 expression also produced near-significant results in univariate survival analysis. Analyses on the effects of p27 in endometrial cancer have produced mixed results in previous studies, thus remaining unclear whether high p27 expression is a poor or a favorable prognostic factor. Further large-scale studies are required to assess the effects of cyclins and p27 in endometrial cancer.

\section{Acknowledgements}

The Authors thank Mr. Risto Bloigu, MSc, for his assistance in the execution and evaluation of the survival analyses. They also thank laboratory technicians Mrs. Erja Tomperi and Mrs. Mirja Vahera.

\section{References}

1 Harashima H, Dissmeyer N and Schnittger A: Cell cycle control across the eukaryotic kingdom. Trends Cell Biol 23(7): 345-356, 2013.

2 Malumbres M and Barbacid M: Cell cycle, CDKs and cancer: A changing paradigm. Nat Rev Cancer 9(3): 153-166, 2009.

3 Abukhdeir AM and Park BH: p21 and p27: Roles in carcinogenesis and drug resistance. Expert Rev Mol Med 10: e19, 2008

4 Besson A, Dowdy SF and Roberts JM: CDK inhibitors: Cell cycle regulators and beyond. Dev Cell 14(2): 159-169, 2008.

5 Finnish Cancer Registry: Cancer Statistics [Internet]. Cited: October 1, 2014. Available from: http://www.cancer.fi/ syoparekisteri/ en/statistics/.

6 Murali R, Soslow RA and Weigelt B: Classification of endometrial carcinoma: More than two types. Lancet Oncol 15(7): e268-e278, 2014.

7 Creasman WT, Odicino F, Maisonneuve P, Quinn MA, Beller U, Benedet JL, Heintz AP, Ngan HY and Pecorelli S: Carcinoma of the corpus uteri. FIGO 26th annual report on the results of treatment in gynecological cancer. Intl J Gyn Obst 95(Suppl 1): S105-S143, 2006.

8 Santala S, Talvensaari-Mattila A, Soini Y, Honkavuori-Toivola M and Santala M: High expression of cyclin A is associated with poor prognosis in endometrial endometrioid adenocarcinoma. Tumor Biol 35: 5395-5399, 2014.

9 Santala S, Talvensaari-Mattila A, Soini Y and Santala M: Prognostic value of cyclin B in endometrial endometrioid adenocarcinoma. Tumor Biol 36: 953-957, 2014.

10 Santala S, Talvensaari-Mattila A, Soini Y and Santala M: Cyclin E expression correlates with cancer-specific survival in endometrial endometrioid adenocarcinoma. Anticancer Res 35(6): 3393-3397, 2015.

11 Chu IM, Hengst L and Slingerland JM: The Cdk inhibitor p27 in human cancer: Prognostic potential and relevance to anticancer therapy. Nat Rev Cancer 8(4): 253-267, 2008.

12 Nycum LR, Smith LM, Farley JH, Kost ER, Method MW and Birrer MJ: The role of p27 in endometrial carcinoma. Gynecol Oncol 81(2): 242-246, 2001.

13 Dellas A, Jundt G, Sartorius G, Schneider M and Moch H: Combined PTEN and p27kip1 protein expression patterns are associated with obesity and prognosis in endometrial carcinomas. Clin Cancer Res 15(7): 2456-2462, 2009.

14 Masciullo V, Susini T, Zamparelli A, Bovicelli A, Minimo C, Massi D, Taddei G, Maggiano N, De Iaco P, Ceccaroni M, Bovicelli L, Amunni G, Mancuso S, Scambia G and Giordano A: Frequent loss of expression of the cyclin-dependent kinase inhibitor p27(Kip1) in estrogen-related endometrial adenocarcinomas. Clin Cancer Res 9(14): 5332-5338, 2003.

15 Shih HC, Shiozawa T, Kato K, Imai T, Miyamoto T, Uchikawa J, Nikaido T and Konishi I: Immunohistochemical expression of cyclins, cyclin-dependent kinases, tumor-suppressor gene products, $\mathrm{Ki}-67$, and sex steroid receptors in endometrial carcinoma: positive staining for cyclin $\mathrm{A}$ as a poor prognostic indicator. Hum Pathol 34(5): 471-478, 2003.

16 Seeber LM, Horree N, van der Groep P, van der Wall E, Verheijen RH and van Diest PJ: Necrosis related HIF-1alpha expression predicts prognosis in patients with endometrioid endometrial carcinoma. BMC Cancer 10: 307-2407-10-307, 2010.

17 Steinbakk A, Skaland I, Gudlaugsson E, Janssen EA, Kjellevold KH, Klos J, Lovslett K, Fiane B and Baak JP: The prognostic value of molecular biomarkers in tissue removed by curettage from FIGO stage 1 and 2 endometrioid type endometrial cancer. Am J Obstet Gynecol 200(1): 78.e1-78.e8, 2009.

18 Steinbakk A, Malpica A, Slewa A, Skaland I, Gudlaugsson E, Janssen EA, Lovslett K, Fiane B, Kruse AJ, Feng W, Yinhua Y and Baak JP: Biomarkers and microsatellite instability analysis of curettings can predict the behavior of FIGO stage I endometrial endometrioid adenocarcinoma. Mod Pathol 24(9): 1262-1271, 2011.

19 Horree N, van Diest PJ, van der Groep P, Sie-Go DM and Heintz AP: Progressive derailment of cell cycle regulators in endometrial carcinogenesis. J Clin Pathol 61(1): 36-42, 2008.

20 Kato N, Watanabe J, Jobo T, Nishimura Y, Fujisawa T, Kamata $\mathrm{Y}$ and Kuramoto $\mathrm{H}$ : Immunohistochemical expression of cyclin $\mathrm{E}$ in endometrial adenocarcinoma (endometrioid type) and its clinicopathological significance. J Cancer Res Clin Oncol 129(4): 222-226, 2003.

21 Oshita T, Shigemasa K, Nagai N and Ohama K: p27, cyclin E, and CDK2 expression in normal and cancerous endometrium. Int J Oncol 21(4): 737-743, 2002.

22 Kuttler F, Valnet-Rabier MB, Angonin R, Ferrand C, Deconinck E, Mougin C, Cahn JY and Fest T: Relationship between expression of genes involved in cell cycle control and apoptosis in diffuse large B cell lymphoma: A preferential survivin-cyclin B link. Leukemia 16(4): 726-735, 2002.

23 Aaltonen K, Amini RM, Heikkila P, Aittomaki K, Tamminen A, Nevanlinna $\mathrm{H}$ and Blomqvist $\mathrm{C}$ : High cyclin $\mathrm{B} 1$ expression is associated with poor survival in breast cancer. $\mathrm{Br} \mathrm{J}$ Cancer 100(7): 1055-1060, 2009.

24 Allan K, Jordan RC, Ang LC, Taylor M and Young B: Overexpression of cyclin A and cyclin B1 proteins in astrocytomas. Arch Pathol Lab Med 124(2): 216-220, 2000.

25 Fung TK and Poon RY: A roller coaster ride with the mitotic cyclins. Semin Cell Dev Biol 16(3): 335-342, 2005.

26 Watanabe J, Sato H, Kanai T, Kamata Y, Jobo T, Hata H, Fujisawa T, Ohno E, Kameya T and Kuramoto H: Paradoxical expression of cell cycle inhibitor p27 in endometrioid adenocarcinoma of the uterine corpus - correlation with 
proliferation and clinicopathological parameters. Br J Cancer 87(1): 81-85, 2002.

27 Akiyama-Abe A, Minaguchi T, Nakamura Y, Michikami H, Shikama A, Nakao S, Sakurai M, Ochi H, Onuki M, Matsumoto K, Satoh T, Oki A and Yoshikawa H: Loss of PTEN expression is an independent predictor of favorable survival in endometrial carcinomas. Br J Cancer 109(6): 17031710, 2013.

28 An HJ, Lee YH, Cho NH, Shim JY, Kim JY, Lee C and Kim SJ: Alteration of PTEN expression in endometrial carcinoma is associated with down-regulation of cyclin-dependent kinase inhibitor, p27. Histopathology 41(5): 437-445, 2002.

29 Morneau M, Foster W, Lalancette M, Van Nguyen-Huynh T, Renaud MC, Samouelian V, Letarte N, Comite de l'evolution des pratiques en oncologie, Comite de l'evolution des pratiques en oncologie, Almanric K, Boily G, Bouchard P, Boulanger J, Cournoyer G, Couture F, Gervais N, Goulet S, Guay MP, Kavanagh M, Lemieux J, Lesperance B, Letarte N, Morneau M, Ouellet JF, Pineau G, Rajan R, Roy I, Samson B, Sideris L and Vincent F: Adjuvant treatment for endometrial cancer: Literature review and recommendations by the Comite de l'evolution des pratiques en oncologie (CEPO). Gynecol Oncol 131(1): 231-240, 2013.

Received September 12, 2016

Revised September 25, 2016

Accepted September 26, 2016 